\title{
Reflets
}

Revue ontaroise d'intervention sociale et communautaire

\section{Les défis de l'intervention interculturelle en milieu minoritaire de langue française en Ontario}

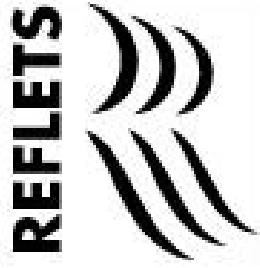

\section{Michèle Kérisit}

Volume 4, numéro 1, printemps 1998

Intervention en contextes minoritaires

URI : https://id.erudit.org/iderudit/026198ar

DOI : https://doi.org/10.7202/026198ar

Aller au sommaire du numéro

Éditeur(s)

Reflets : Revue ontaroise d'intervention sociale et communautaire

ISSN

1203-4576 (imprimé)

1712-8498 (numérique)

Découvrir la revue

Citer cet article

Kérisit, M. (1998). Les défis de l'intervention interculturelle en milieu minoritaire de langue française en Ontario. Reflets, 4(1), 75-99.

https://doi.org/10.7202/026198ar

\section{Résumé de l'article}

Cet article explore les tensions qui président à l'insertion des nouveaux arrivants de languefrançaise dans la communauté franco-ontarienne et leur impact sur la façon dont on peutaborder l'intervention interculturelle en milieu minoritaire francophone en Ontario. Les défisde l'intervention naissent d'une part d'une logique politique forgée dans l'histoire des relationsentre anglophones et francophones nés au Canada, logique sur laquelle les nouveaux arrivantsont peu de prise; d'autre part, une logique proprement identitaire qui tend à créer des "forteresses»difficilement accessibles aux nouveaux venus. Pourtant, selon l'auteure, nous sommes tousmultiples et devrions utiliser la multiplicité de nos identités - en particulier notre expériencede minoritaires afin de créer des ponts.
Tous droits réservés $(\subset$ Reflets : Revue ontaroise d'intervention sociale et communautaire, 1998
Ce document est protégé par la loi sur le droit d'auteur. L'utilisation des services d’Érudit (y compris la reproduction) est assujettie à sa politique d'utilisation que vous pouvez consulter en ligne.

https://apropos.erudit.org/fr/usagers/politique-dutilisation/ 


\section{Les défis de l'intervention inter- culturelle en milieu minoritaire de langue française en Ontario ${ }^{1}$}

Cet article explore les tensions qui président à l'insertion des nouveaux arrivants de langue française dans la communauté franco-ontarienne et leur impact sur la façon dont on peut aborder l'intervention interculturelle en milieu minoritaire francophone en Ontario. Les défis de l'intervention naissent d'une part d'une logique politique forgée dans l'histoire des relations entre anglophones et francophones nés au Canada, logique sur laquelle les nouveaux arrivants ont peu de prise; d'autre part, une logique proprement identitaire qui tend à créer des «forteresses» difficilement accessibles aux nouveaux venus. Pourtant, selon l'auteure, nous sommes tous multiples et devrions utiliser la multiplicité de nos identités — en particulier notre expérience de minoritaires - afin de créer des ponts.

\section{Michèle Kérisit}

Professeure, École de service social, Université d'Ottawa

Pour sa part, le concept de minorité ne renvoie pas à l'ethnie mais plutôt au rapport de pouvoir qui fait que l'on se perçoit comme un membre de groupe minoritaire. Ainsi, lorsque l'on parle de groupe minoritaire, il n'est pas question de nombre mais d'abord et avant tout de pouvoir. Le concept de minorité a ainsi une connotation politique que la notion d'ethnicité n'a pas, parce qu'elle renvoie strictement à des caractéristiques objectives et non au rapprt de pouvoir qui fonde son existence (Cardinal 1997: 156).

Cet article propose une réflexion sur les particularités de l'intervention interculturelle en milieu minoritaire francophone 
"...on accorde généralement peu d'attention aux interventions menées dans un contexte de hiérarchisation des rapports sociaux interethniques, entre groupes minorisés qui se retrouvent placés à différents niveaux de l'échiquier sociopolitique.» en Ontario. Les questions qui y sont soulevées sont nées d'une certaine insatisfaction devant la façon dont sont traitées les questions d'interculturalité dans les écrits portant sur la formation en intervention interculturelle et de mon expérience de formatrice auprès d'un public d'intervenantes et d'intervenants francophones en Ontario. En effet, la plupart des écrits sur l'intervention interculturelle, qu'ils viennent du Québec (Jacob 1992; Legault 1993; Legault et Lafrenière 1992; Barrette, Gaudet et Lemay 1993), du Canada de langue anglaise (Estable, Mayer et Pon 1997), de France (Cohen-Émerique 1993), de Grande-Bretagne (Dominelli 1988) ou des États-Unis (Ridley 1995; Locke 1992), présupposent que les relations d'intervention prennent place dans un contexte où les rapports sociaux s'élaborent entre groupe «majoritaire» (dont sont en général issus les intervenants et les intervenantes) et groupes «minoritaires» (en général composés d'une clientèle immigrante ou réfugiée). Au Canada et au Québec, l'essence de la formation en intervention interculturelle consiste alors, et avec raison, à faire prendre conscience aux intervenants et intervenantes (le plus souvent blancs, d'origine européenne, et nés au Canada) des différentes dimensions du pouvoir qu'exerce le groupe dominant auquel ils appartiennent sur l'expérience de vie des groupes «minoritaires» (les «immigrants», les «Noirs» les «minorités visibles», les «ethnoculturels») 2 .

Intervenants et intervenantes sont invités à se questionner face à cette position de pouvoir afin de mieux comprendre la complexité des situations vécues par leur clientèle. Au mieux, une telle formation les confronte avec leurs propres préjugés et les incite à comprendre comment le pouvoir de la société d'accueil, dont ils sont les représentants, se naturalise, s'exprime et s'exerce à travers eux. Par contre, on accorde généralement peu d'attention aux interventions menées dans un contexte de hiérarchisation des rapports sociaux interethniques, entre groupes minorisés qui se retrouvent placés à différents niveaux de l'échiquier sociopolitique. Pourtant, en cette fin de siècle, il est important que s'élabore une réflexion sur les pratiques interculturelles en tenant compte de la multiplicité et de la fragmentation des positionnements identitaires et politiques. 
Cet article vise donc à recadrer, en les contextualisant, les pratiques d'intervention interculturelle auprès d'immigrants et de réfugiés d'expression française par des intervenants et intervenantes francophones de l'Ontario. Pour améliorer ces pratiques, il importe d'abord d'expliciter de façon très claire les enjeux inscrits dans la dynamique de minorisation des groupes au Canada. Les rapports sociaux et de pouvoir y sont en effet codifiés en fonction de valeurs, de lois et de politiques, d'une histoire spécifique de l'immigration et des rapports entre les deux "peuples fondateurs", de langue anglaise et de langue française. Ces codes obéissent à des logiques politiques, identitaires, économiques et légales dont certaines sont communes à l'ensemble du Canada et d'autres plus spécifiques aux francophones qui vivent à l'extérieur du Québec.

Cet article ne prétend pas traiter de l'ensemble des problématiques rencontrées par les nouveaux arrivants et du soutien apporté ou non par les intervenants sociaux. Il existe un nombre non négligeable d'ouvrages traitant des multiples facettes de l'intégration: intégration à l'emploi (Daenzer 1991; Berger 1996), situation spécifique des femmes (Groupe de travail sur les femmes immigrantes et la violence conjugale 1997; Vatz Laaroussi 1996; McLeod et Shin 1994; Boyd 1991; Labelle 1990), apprentissage des langues, problématiques familiales et accès aux services (Heneman 1994; Kérisit, à paraitre), problématiques de santé (Masi 1993; Clarkson et Dahan 1997; Clarkson et Eustache 1997, Clarkson et Tran The Nghi 1997) et de santé mentale (Bibeau et al. 1992), de racisme (McKague 1991). Ces ouvrages contiennent des éléments qui peuvent s'appliquer à la situation des immigrants et des réfugiés francophones en Ontario qui font face à des difficultés d'insertion à l'emploi, d'accès aux services et à des conditions de vie marquées par l'isolement. Notre but est plutôt de démêler quelques écheveaux afin de proposer un cadre d'analyse et de lancer la discussion sur les rapports entre nouveaux arrivants de langue française et francophones installés depuis longtemps en Ontario, cadre d'analyse qui a été très peu abordé directement dans les écrits, jusqu'à date.

Pour cela, nous articulerons notre analyse autour des questions suivantes: Quelles sont les logiques qui régissent et traversent les 
rapports entre nouveaux arrivants et anciens arrivants de langue française en Ontario? Quelles sont les conséquences de ces logiques sur l'intervention et comment peut-on les prendre en considération dans la pratique?

La première section fera le point sur certains éléments des logiques politiques qui traversent les rapports entre immigrants et non-immigrants de langue française, alors que la seconde cherchera à comprendre les logiques proprement culturelles et identitaires qui colorent ces rapports.

\section{Logiques politiques}

Les termes utilisés pour désigner et dénombrer ceux et celles qui sont perçus comme «autres» ou «différents» ou «venus d'ailleurs» sont importants. Ces termes sont révélateurs des différents positionnements des populations récemment arrivées sur l'échiquier politique; ils leur conferent une identité, un nom qui les poursuit parfois malgré eux ${ }^{3}$. La manière de dénombrer les nouveaux et anciens arrivants au Canada donne aussi des pistes sur la façon dont ils sont placés sur l'échiquier politique. Cet exercice de déchiffrement nous conduira à examiner les conséquences pour le domaine de l'intervention pour parvenir à mieux comprendre les dynamiques sous-jacentes.

\section{Les chiffres}

Il est d'usage, quand on veut faire le point au sujet d'une question comme l'intervention interculturelle, de dresser un portrait démographique des groupes en présence. Cependant, puisqu'il n'existe pas de relevé statistique fiable des nouveaux Ontariens s'exprimant en français, nous devons faire de la conjecture. L'ACFO (Gilbert et Langlois 1994) propose le chiffre de 82000 
personnes mais ajoute qu'un tel chiffre n'est qu'«une approximation fort imparfaite du nombre d'ethnoculturels francophones qui participent au développement de la communauté francoontarienne» (1994: 54). Basant leur estimation sur le croisement de données du recensement de 1991, les auteurs relèvent l'impossibilité d'inclure dans leur relevé les francophones «qui, sans avoir le français comme langue maternelle, l'utiliseraient néanmoins dans la vie quotidienne» (idem: 54). Les chiffres se réduisent encore si l'on se restreint à ne compter que les personne dont le français est la langue parlée à la maison, soit 48710 personnes.

Les chercheurs disposent en effet de peu de variables pour se faire une idée plus juste de la population ethnoculturelle francophone de l'Ontario. D'abord, la personne a le français comme langue maternelle (réponse unique ou multiple) ou parle le français à la maison (réponse unique ou multiple); ces deux variables, combinées avec une origine ethnique autre que française ou britannique, forment la base de recensement a posteriori de la population francophone ontarienne qui n'est pas née au Canada. Un décompte supplémentaire permet également de distinguer première génération d'immigration et personnes nées au Canada. Pour avoir une idée simplifiée de la population immigrante et réfugiée francophone, il s'agit donc de combiner la variable «née hors du Canada» avec les combinaisons évoquées plus haut. En partant de la variable «langue maternelle», nous aboutissons au total d'environ 25000 personnes. Si l'on ne décompte que celles dont le français est la langue parlée à la maison, c'est le chiffre de 18000 personnes qui s'impose (Gilbert et Langlois 1994: 55).

"...les nouveaux arrivants d'expression française, qui n'ont le français ni comme langue maternelle ni comme langue parlée à la maison, ne se trouvent pas recensés comme francophones.»
Notons que dans les deux cas, les nouveaux arrivants d'expression française, qui n'ont le français ni comme langue maternelle ni comme langue parlée à la maison, ne se trouvent pas recensés comme francophones.

Les chiffres de 25000 ou de 18000 personnes sous-estiment donc grandement la présence de nouveaux arrivants de langue française, faisant ainsi disparaître de la carte de la francophonie bon nombre de personnes qui pourraient venir grossir les rangs des Franco-Ontariens. En fait, nous proposons que ce flou dans 
le recensement fait partie d'une logique politique de la désignation, qui est elle-même le produit d'une histoire et d'une dynamique politique propres aux situations de minorisation des francophones en Ontario.

Plutôt que d'essayer de faire des contorsions statistiques, il est sans doute préférable de questionner les raisons qui conduisent à vouloir dénombrer les populations. Nous savons bien qu'une communauté francophone hors Québec ou franco-ontarienne n'existe, pour l'État, que «là où le nombre le justifie». Est-ce à dire que les gens n'existent pas s'ils ne sont pas suffisemment nombreux? Si on sait qu'il n'y a pas plus de 15, 356, 2000 ou 40000 immigrants de langue française dans tel secteur donné, il ne vaudrait donc pas la peine de s'en faire? Du point de vue de la rationalité bureaucratique et politique du système, une telle démarche a un sens. Par contre, du point de vue de la personne qui veut avoir accès à des intervenants qui la comprennent, la logique du nombre n'a pas grand sens et remet en question la question du droit de se faire servir en français pour tous les francophones de l'Ontario.

Cependant, lorsque cette logique des chiffres imprègne la culture des intervenants francophones nés au Canada, cela devient inquiétant. Les chiffres, sous leurs airs objectifs, cachent souvent des calculs savants qui échappent aux citoyens. Ils permettent de mettre en place une planification de services répondant à la majorité mais ne prenant pas en compte les besoins de la minorité. Les Franco-Ontariens nés ici connaissent le «truc», eux qui ont souvent vu leur passer sous le nez un certain nombre de services, parce que «leur nombre ne le justifiait pas». On pourrait donc se demander s'il est judicieux de recommencer ce même petit jeu avec les nouveaux arrivants, au sein même de la communauté ontarienne française de souche, car il arrive bien souvent d'entendre dire: «mais ils ne sont pas si nombreux que cela!»... ou «ils préferrent parler anglais», ou «ils ne viennent pas chez nous» ou encore «il n'y en a pas ici».

Une logique dominante gouverne donc les données dont nous disposons quant aux minorités faisant partie de la francophonie ontarienne. Cette logique est avant tout politique, au sens large 
du terme, dans la mesure où elle oblige les personnes et les groupes à s'aligner sur des problématiques linguistiques dont ils ne sont pas nécessairement porteurs mais qui sont encadrées par des politiques dont nous allons toucher mot dans les pages suivantes.

\section{La question de la langue}

À cette question des chiffres, s'ajoutent évidemment le flou et les controverses qui entourent le débat sur la langue maternelle et la langue d'usage, qui sert de toile de fond pour déterminer qui est francophone ou ne l'est pas... Qu'arrive-t-il si le français a été acquis sur le tard, à travers un sytème éducatif, par exemple? ou si le français a été parlé comme langue de commerce mais ne représente pas une langue dans laquelle on a l'habitude de parler d'émotions, de sentiments, de relations sociales? Est-ce que «ces gens-là» sont francophones? ${ }^{4}$ Une vision de la francophonie basée exclusivement sur la défense de la langue peut-elle rassembler des Franco-Ontariens (avec leur passé de lutte pour le maintien $\mathrm{du}$ français) et des nouveaux venus qui ne ressentent pas nécessairement le même attachement à cette langue, ou du moins pas pour les mêmes raisons et pas de la même façon? Cette question importante dérange, car elle traduit une différence de positionnement vis-à-vis de la défense des droits linguistiques des francophones en Ontario. Or ce positionnement implique, des deux côtés, une histoire et une volonté de survie qui ne sont pas nécessairement les mêmes. Il est aussi traversé par des problématiques, du moins pour les nouveaux arrivants, qui relèvent de la survie économique pure et simple, dans la mesure où c'est dans leurs rangs que l'on retrouve sans doute le plus de gens «unilingues» francophones (indépendamment du fait que plusieurs parlent déjà d'autres langues que le français ou l'anglais). La défense des droits des francophones ne se vit donc pas de la même façon que l'on soit établi en Ontario depuis très longtemps ou très récemment. Dans ce débat, souvent joué de façon implicite, se retrouvent cependant les grands cadres de politiques qui façonnent la scène canadienne depuis trente ans: politiques de multiculturalisme et de bilinguisme 
"L'immigration au Canada, surtout depuis les dix dernières années, ne peut se comprendre seulement à l'échelle du Canada ou de l'Ontario. Pour la comprendre, il faut absolument remettre la situation dans son contexte global et mondial, géopolitique.» ont en quelque sorte imprégné la façon dont les questions sont abordées.Voyons ce qu'il en est plus en détails.

L'immigration au Canada, surtout depuis les dix dernières années, ne peut se comprendre seulement à l'échelle du Canada ou de l'Ontario. Pour la comprendre, il faut absolument remettre la situation dans son contexte global et mondial, géopolitique. Certaines personnes viennent par "choix» au Canada - encore que l'on puisse se demander en quoi consiste ce choix. C'est encore la majorité. D'autres, moins nombreuses, passent à travers les divers processus qui leur permettent d'obtenir le statut de réfugié, puis de résidents permanents. Jusque dans les annés 70, la majorité des immigrants venait de pays occidentaux, mais depuis ils arrivent majoritairement des pays du Sud (Badets 1993). Nous avons tous entendu parler de certains immigrants venant de Hong Kong, favorisés par une politique d'immigration favorable aux investisseurs et hommes d'affaires. Il y a peu de chance que les travailleurs sociaux les retrouvent dans leurs bureaux. La plupart des personnes qui doivent demander de l'aide sont celles qui ont vécu dans des pays déchirés par la pauvreté et ou la guerre, même si elles-mêmes avaient déjà les moyens de partir. La majorité silencieuse la plus malheureuse - à $80 \%$ constituée de femmes et d'enfants - se retrouve dans des camps de réfugiés dont on nous donne à voir l'horreur à la télévision. Ceux-là non plus ne se retrouvent pas dans les bureaux des services sociaux.

Bref,la situation des nouveaux pays d'émigration a une histoire: celle du colonialisme, puis des mouvements de libération contre l'impérialisme, puis, pour résumer, la plongée dans la pauvreté grâce surtout à ce qu'on a appelé la restructuration de la dette, menée par la Banque mondiale et le Fonds monétaire international, gouvernés par des intérêts occidentaux. Cette histoire est aussi faite d'intervention militaire par certains pays, comme la France ou les États-Unis, ou l'ancienne URSS, par la corruption d'élites dirigeantes et dictatoriales au service d'intérêts étrangers. L'histoire collective des nouveaux arrivants n'est pas faite d'une connaissance «bénigne», «gentille» de l'aide humanitaire. Elle est pleine de fureur et de mort, de misère et d'indignité. 
Si nous insistons sur cela, c'est que cette histoire a une grande importance pour comprendre la situation des nouveaux arrivants francophones au Canada et en Ontario. Ceux-ci ont eu souvent un rapport compliqué avec des pays colonisateurs de langue française (la France pour l'Afrique du Nord - Algérie, Maroc, Tunisie - et de l'Ouest, le Vietnam, le Laos et le Cambodge, la Somalie par l'intermédiaire de Djibouti, le Liban; la Belgique, mais aussi la France, pour le Burundi, le Rwanda et la République démocratique du Congo, l'exemple Zaïre). Ils ont, à un moment ou un autre, été éduqués dans des institutions françaises installées dans leur pays ou dans les pays colonisateurs.

D'une part, parler français n'est donc pas nécessairement un acte de «rébellion» vis-à-vis une société dominante parlant anglais; au contraire, cela peut être parler la langue de celui qui vous a dépossédé de votre identité maternelle. D'autre part, parler français signifie aussi la possibilité d'accéder à un niveau de vie meilleur, grâce aux études et à l'insertion dans une société dominante, de langue française dans ce cas. De nombreux immigrants de langue

"L'apprentissage ou le maniement d'une langue - que ce soit le français ou l'anglais sont donc souvent perçus comme un moyen, non comme une fin ou un signe d'attachement à une histoire et à des droits fondés dans l'histoire d'un groupe.» française ont en effet un niveau de scolarité élevé. L'apprentissage ou le maniement d'une langue — que ce soit le français ou l'anglais - sont donc souvent perçus comme un moyen, non comme une fin ou un signe d'attachement à une histoire et à des droits fondés dans l'histoire d'un groupe.

À cette histoire propre à de nombreux nouveaux arrivants francophones en Ontario, nous ajouterons le cadre des politiques de multiculturalisme, créé il y a déjà plus d'un quart de siècle au Canada, cadre officiel dans lequel s'insère la francophonie ontarienne et qui encapsule les relations entre citoyens canadiens de souche et citoyens canadiens nés ailleurs. Cette politique est née sur les talons de la Commission sur le Bilinguisme et Biculturalisme de 1967 qui tentait de "faire le point» sur les relations ethniques entre Canadiens d'origine britannique et ceux d'origine française. Elle a été perçue comme une volonté de «noyer le poisson», en mettant sur un même plan les relations historiques de domination anglo-française et les autres groupes ethniques. Selon cette logique, la légitimité des revendications canadiennes françaises perdrait de sa force en apparaissant "parallèle» à celles, par exemple, des 
"Le bilinguisme officiel promu par les instances canadiennes... et les droits francophones inscrits dans la Loi 8 de l'Ontario... sont une façade que les nouveaux arrivants francophones «unilingues» ne tardent pas à percer, le plus souvent à leurs propres frais.»
Ukrainiens ou des Italiens installés au pays. Ce n'est pas mon but, ici, de discuter de cette controverse, mais de signaler combien elle entache encore les relations entre nouveaux arrivants et Canadiens français de souche, surtout avec ceux qui ont opté pour l'anglais comme langue de communication.

L'objectif officiel de la politique de multiculturalisme était de permettre aux communautés d'origine non-britannique et nonfrançaise de se développer des outils d'ancrage dans la société canadienne sans pour autant perdre leur "culture». Je sais qu'il est de bon ton actuellement de critiquer cette politique. Il me semble pourtant qu'une telle politique a du bon. Souvent décriée comme fossilisatrice de cultures et coupable de ghettoïser les gens, cette politique, quoiqu'on en dise, permet officiellement à bien des groupes de nouveaux arrivants de se créer des points de repère dans un monde étranger. Les organismes subventionnés par les instances d'État (en particulier fédérales) chargées du multiculturalisme permettent d'atténuer les pertes vécues par le fait même d'émigrer et de recréer, même momentanément et artificiellement, des instants où l'on peut se réconcilier avec ses souvenirs.

Le bilinguisme officiel promu par les instances canadiennes (le gouvernement fédéral est responsable de l'immigration) et les droits francophones inscrits dans la Loi 8 de l'Ontario (Loi sur les services en français) sont une façade que les nouveaux arrivants francophones «unilingues» ne tardent pas à percer, le plus souvent à leurs propres frais. Il est, en effet, pratiquement impossible de trouver un emploi et même de recevoir des services si l'on ne parle pas l'anglais en Ontario.

Choix de langue d'insertion, participation ou non aux combats pour la défense des minorités de langue française au Canada ou pour le développement de services en français en Ontario prennent donc une couleur toute autre si on les regarde dans une perspective propre à ceux et celles qui s'installent en Ontario. L'intégration des nouveaux arrivants dans les communautés franco-ontariennes est porteuse de très grandes tensions dans la mesure où s'y posent, comme en reflet, les enjeux propres à l'intégration des minorités francophones vivant à l'extérieur du Québec. Il est évidemment 
tentant de souligner les intérêts objectifs communs entre communautés «de souche» et communautés nouvellement installées. C'est d'ailleurs ce que font plusieurs personnes dans le milieu franco-ontarien, estimant que l'intégration des immigrants et réfugiés de langue française dans une communauté francoontarienne permettrait de renforcer la présence du français en Ontario et de parer au spectre de l'assimilation et de la disparition. Cette pratique contribue cependant à nier l'histoire des groupes en présence et les dimensions culturelles et identitaires qui pèsent de leur poids sur l'insertion des nouveaux arrivants dans une société d'accueil francophone qui elle aussi a son histoire, ses dimensions culturelles et identitaires. Ce sont ces dernières dimensions que nous nous attacherons à développer dans la section suivante.

\section{Logiques culturelles et identitaires}

"La différence entre les cultures est souvent nommée comme l'obstacle principal d'une relation harmonieuse entre intervenants et clients. Nous examinerons de plus près cet argument afin de voir en quoi, précisément, il consiste.»
Si la logique qui domine les rapports entre minorité francoontarienne née ici et immigrants francophones relève des grands cadres politiques qui construisent le débat propre au Canada, cela ne signifie pas que l'on doive écarter d'autres élements qui ont autant de force dans l'expérience quotidienne des contacts entre les gens. Le premier de ces éléments, souvent soulevé par des intervenantes et des intervenants travaillant auprès des «immigrants», concerne la culture. La différence entre les cultures est souvent nommée comme l'obstacle principal d'une relation harmonieuse entre intervenants et clients. Nous examinerons de plus près cet argument afin de voir en quoi, précisément, il consiste. Une telle démarche nous semble importante dans la mesure où elle pose la question de l'identité, cruciale à la fois pour ceux et celles qui immigrent au Canada et pour ceux et celles qui appartiennent à la minorité de langue française née ici. 


\section{Immigrant ou émigrant?}

"Toute immigration suppose un mouvement inverse à l'im-migration, qui est celui de l'émigration.»
«En gardant sous silence l'acte d'émigration, nous nous fermons les yeux à ce qui, justement, constitue la réalité des «immigrants».»
Le terme le plus fréquemment utilisé pour désigner les personnes qui viennent s'installer au Canada est sans doute celui d'immigrant. Or que signifie immigrer? Toute immigration suppose un mouvement inverse à l'im-migration, qui est celui de l'é-migration.Voici comment Moro (1993:162) exprime ce double mouvement dont nous verrons ensuite les conséquences :

[migrer, c'est] émigrer, quitter, perdre l'enveloppe des lieux, des sons, d'odeurs, de sensations de toutes sortes qui constituent les premières empreintes sur lesquelles s'est établi le codage du fonctionnement psychique;

Migrer c'est aussi immigrer, c'est à dire reconstruire seul, en l'espace de quelques années, ce que des générations ont lentement élaboré et transmis.

Or la littérature sur l'intervention auprès des immigrantes et des immigrants est majoritairement porteuse d'images relevant du deuxième volet de cet acte de «migrer», c'est-à-dire l'installation en pays d'accueil (Bertot \& Jacob 1991; Heneman et al. 1994). Si elle fait référence aux situations pré-migratoires, c'est souvent pour souligner la nécessité de prendre en compte les traumatismes vécus par les réfugiés (Beiser 1988; Mills 1993, Hyman, Beiser et NhiVu 1996). En général, cependant, mettre en oeuvre et penser l'intervention interculturelle auprès des «immigrants» se résume à faciliter l'acte de «reconstruction solitaire» dont parle Moro, au sein d'une société d'accueil présumée fixe. En gardant sous silence l'acte d'émigration, nous nous fermons les yeux à ce qui,justement, constitue la réalité des «immigrants». Entre départ (forcé ou non) et intégration (forcée ou non), il existe un présent où s'inscrit la vie de la personne et de sa famille, entre nostalgie et espoir. On ne perd «l'enveloppe des lieux» qu'en fonction d'un projet, dans lequel on n'est plus tout à fait celui ou celle qu'on était et qui définit plus ou moins clairement qui on sera. Immigrer, c'est donc gérer des identités multiples, qui se dessinent en fonction du projet de «réussir» en pays d'accueil. 
"Une intervention qui nie ou oublie la question de l'émigration - et donc du parcours de l'émigrant - revient, à notre avis, à rejeter dans un présent problématique, des difficultés qui relèvent $d u$ sens que l'on peut donner à l'un des actes "centraux» de son existence, celui de s'être dissocié de l'enveloppe de sens construite patiemment par les générations qui nous ont précédés,...»
Parler «d'immigrants» revient aussi à se poser la question suivante : quand cesse-t-on d'être un immigrant? Est-ce quand on a perdu le souvenir du "pays», quand la famille restée au pays a disparu? Quand on est né au Canada et que l'on a suivi le cursus de l'enfance canadienne mais que les mythologies familiales sont encore présentes? Est-ce seulement la seconde génération (les petits enfants de ceux qui ont migré) qui peut se dire nonimmigrante? Que faire alors des photos retrouvées, du nom que l'on porte?

Ces questions sembleraient dénuées de sens si nous ne prenions en considération le premier mouvement de toute migration qui est celui de se dé-partir du sens que l'on donne à un environnement physique, social, psychologique et spirituel, qu'il ait un pendant dans la réalité du pays quitté, qu'il ait été transformé par l'histoire du pays d'origine ou qu'il n'existe que dans son propre imaginaire. Ce sens constitue, avec autant de force que tous les efforts d'intégration, la réalité de la migration. Une intervention qui nie ou oublie la question de l'émigration - et donc du parcours de l'émigrant — revient, à notre avis, à rejeter dans un présent problématique, des difficultés qui relèvent du sens que l'on peut donner à l'un des actes "centraux» de son existence, celui de s'être dissocié de l'enveloppe de sens construite patiemment par les générations qui nous ont précédés, ce que l'on appelle la culture et qui forge notre identité. Immigrer, c'est avoir changé dans son rapport identitaire avec ceux qui sont restés au pays, en particulier les membres de sa famille.

Or nous avons tous une enveloppe de sens avec des lieux, des sons, des odeurs de mémoire. La différence entre immigrants et non immigrants n'est pas tant dans la qualité et la nature de la culture dont ils ou elles sont porteurs (bien qu'elle ait son importance, nous le verrons) que dans le fait que les premiers ont quitté certains points de repère identitaires qui donnaient sens à certains actes et à certains gestes. À cela nous ajouterons l'exigence d'une conscience aiguë de l'impossibilité, pour l'immigrant, de retrouver sereinement les éléments de la culture dans laquelle il a forgé son identité première. Immigrer, c'est vivre en exil, quoi qu'on en dise. 
«Les Franco-Ontariens nés ici possèdent eux aussi leur «enveloppe de sens" grâce à laquelle ils donnent sens à leurs gestes et aux relations humaines.»

\section{"Un tel mouvement} cependant agit comme un miroir qui réfléchit les normes, les valeurs et les habitudes de celui ou de celle qui intervient. Il réfléchit aussi les insécurités.»
Les Franco-Ontariens nés ici possèdent eux aussi leur «enveloppe de sens» grâce à laquelle ils donnent sens à leurs gestes et aux relations humaines. Ils constituent, au même titre que d'autres groupes, une «tribu» distincte, avec non seulement son imaginaire, ses rituels, ses croyances, ses sons et ses façons de faire mais aussi ses divisions, ses classes et sa propre représentation de son histoire. Ce n'est qu'en étant conscient de sa propre «enveloppe de sens» que l'on arrive à distinguer ce qui nous appartient en tant qu'intervenante ou intervenant et ce qui appartient au client ou à la cliente immigrante. Une certaine dose d'introspection quant à ses propres valeurs et ses propres présupposés, à la façon dont on se pense franco-ontarienne ou franco-ontarien, s'impose donc pour pouvoir intervenir de façon culturellement sensible.

Ce mouvement de retour sur sa propre «enveloppe de sens» se fait obligatoirement par l'immigrante ou l'immigrant mais est souvent absente de la réflexion des membres de la société d'accueil. Pourtant, il est nécessaire pour accueillir ce que «l'étranger» a à dire. Un tel mouvement cependant agit comme un miroir qui réfléchit les normes, les valeurs et les habitudes de celui ou de celle qui intervient. Il réfléchit aussi les insécurités. La question est importante, à mon avis, en contexte minoritaire francoontarien; je donnerai deux exemples pour illustrer cette idée.

\section{«La fragilité de la modernité en nous»}

Certains auteurs (Welch 1995; 1993; Cardinal 1997) ont décrit des préjugés qui courent encore sur les Franco-Ontariens : ceuxci seraient des ruraux peu instruits, à la nombreuse progéniture, soumis à un catholicisme conservateur, etc. Or que signifie une telle caricature dans un milieu nord-américain? Elle est le symbole de la non-modernité, d'une société archaïque qui n'a pas encore accédé au fonctionnement égalitaire, à la technologie, aux idées neuves. Elle permet au segment dominant de la population de justifier son irrespect vis-à-vis d'un autre segment qu'il veut assimiler, exploiter ou rejeter.Telle est la nature même des rapports de minorité à majorité quand ils sont examinés sous l'angle des rapports de pouvoir. Que peut être alors l'une des réactions 
"Être confronté à des membres d'une société que l'on considère archaïque nous oblige à confronter ce que Willy Appolon (1993), parlant du choc des cultures an Québec, appelle la «fragilité de la modernité en nous».» possibles devant d'autres stéréotypes qui se rapprochent dangereusement des stéréotypes dont on est soi-même l'objet? Ne serait-il pas mieux, à tout prendre, de se dissocier de façons de faire qui justement rappellent de trop près les accusations dont on est l'objet? Être confronté à des membres d'une société que l'on considère archaïque nous oblige à confronter ce que Willy Appolon (1993), parlant du choc des cultures au Québec, appelle la «fragilité de la modernité en nous». Par exemple, ce n'est pas un hasard si la question du foulard que portent certaines femmes musulmanes suscite tant de passion dans des sociétés qui se souviennent très bien de leur «non-modernité» dans les relations entre hommes et femmes (le Québec, la France...). Les «démons» qui sont projetés sur ces jeunes femmes ne se trouvent pas nécessairement chez ces dernières (ou alors ils ne sont pas de même nature), ils se trouvent plutôt chez ceux qui les voient comme tels.

La possibilité d'une intervention culturelle passe donc par une réflexion sur ses propres "démons» afin de ne pas les transposer chez les autres. Cela signifie, pour les francophones vivant en situation minoritaire, repenser le rapport qu'ils entretiennent avec un monde à dominance anglo-saxonne. En effet, pour reprendre le thème de la modernité, on peut par exemple se demander comment s'expriment les signes de cette modernité. N'est-ce pas par la langue anglaise (la langue de la technologie, de la science, du droit même, des médias)? Cette «fragilité de la modernité en nous» ne passe pas en fait par une réelle "non-modernité», elle a été serinée depuis un siècle à l'égard des Canadiens français. Cette fragilité est une fragilité politique, elle n'est pas une fragilité de fait. Lire la différence sur le mode de la non-modernité, c'est continuer une logique politique qui justement est discutable. C'est, en demandant aux «autres» de changer pour devenir «plus comme nous», reproduire des façons d'être qui ont justement été combattues depuis longtemps par beaucoup de Franco-Ontariens et Franco-Ontariennes.

\section{Les identités multiples}

La question identitaire n'est pas nouvelle pour nombre de FrancoOntariens. Le débat déjà passé sur la façon de se nommer (Ontarois, 
"...chacun reconnaîtra qu'il ou elle est multiple : notre identité ne coïncide pas nécessairement avec une seule culture, un seul peuple, une seule ethnie.»
Franco-Ontarien, Canadien français) (Juteau 1980) fut l'un des signes de cette volonté de mettre en mot la difficulté de définir qui sont les Franco-Ontariens. Les préoccupations face à l'assimilation ou aux mariages mixtes sont aussi des endroits où s'expriment les questions identitaires sur la place publique. Le deuxième exemple que je voudrais apporter pour illustrer les défis de l'intervention interculturelle en milieu francophone minoritaire concerne justement la façon dont on définit son identité. Dans les paragraphes traitant de la langue, nous avons sous-entendu qu'il était évident que langue et culture, pour les nouveaux arrivants francophones, ne coïncidaient pas. De la même façon, chacun reconnaitra qu'il ou elle est multiple: notre identité ne coïncide pas nécessairement avec une seule culture, un seul peuple, une seule ethnie. Selon la circonstance, dans la vie courante et quotidienne,j'utiliserai ou je me sentirai plus proche des femmes, des francophones, des victimes de l'holocauste, des mères chefs de famille, etc... parce que je suis à la fois, par exemple, femme, francophone, juive et que j'élève mes enfants seule. Nos identités sont labiles; les ambiguïtés, les ambivalences et les passerelles sont le lot de l'humanité; les identités ethniques sont traversées par d'autres identités, de sexe, de religion, d'orientation sexuelle et elles changent en fonction des positionnements politiques et familiaux et avec l'âge, parfois... Bien souvent cette labilité des identités, que connaissent bien des Franco-Ontariens et FrancoOntariennes mais qui fait le désespoir de ceux qui voudraient, par exemple, fixer à tout jamais une identité franco-ontarienne, est en fait une chance pour intervenir de façon sensible dans la relation d'aide interculturelle. L'ambigüité et l'ambivalence, même douloureuses, sont une chance à saisir car elles permettent de comprendre le processus que peut vivre une personne qui émigre (et par conséquent immigre). Plutôt que de tenter de faire coïncider la conscience que l'on a de soi-même avec une identité ethnique unique, franco-ontarienne, par exemple, au risque de rendre rigides les frontières qui existent entre soi et les autres, il vaudrait mieux écouter les multiples identités que l'on possède pour entendre ce qu'on pourrait appeler «l'étranger ou l'étrangère en soi», au risque de perdre un peu du sentiment de sécurité que 
procure l'expression «je suis X» (canadienne française, québécoise, canadienne, ontaroise, marocaine ou berbère). La question qui se pose n'est donc pas «qui suis-je», mais «comment puis-je utiliser mes passeports pour avoir accès au territoire de l'autre?». Comment puis-je utiliser, stratégiquement, l'ensemble des identités que je possède pour vivre ou survivre dans un milieu qui a tendance à rigidifier les frontières, à construire des ghettos et à hiérarchiser les relations humaines en fonction de critères de race, d'ethnie et de culture? Il n'y a pas si longtemps, on parlait encore de «sauvages» et de la «race canadienne-française». De la même façon, l'intervenante ou l'intervenant saura qu'il ou elle n'a pas devant lui ou elle quelqu'un qui est exlusivement «africaine» ou «musulmane» ou «réfugiée». Il ou elle a devant elle une personne qui, à travers le processus d'émigration / immigration défini plus haut et à travers ses multiples allégeances identitaires propres, est à la fois différente et semblable.

Pourtant, pourra-t-on objecter, il est évident que les distances culturelles existent. Ceci est certainement vrai. Mais dans quelle mesure est-ce que ce sont elles qui font la «différence»? Combien de fois un retard a-t-il été mis sur le dos de la "culture» alors qu'en fait ce retard n'était dû qu'à la méconnaissance des horaires d'autobus, ou du quartier? De la même façon, combien de fois n'entendons-nous pas que, si tel ou tel organisme n'a pas, en son sein, de représentation «ethnoculturelle», c'est parce qu' «ils» ne veulent pas?

Cette question de l'intégration des nouveaux arrivants dans les services sociaux francophones, dans l'orientation qu'ils prennent ou dans l'action sociale qu'ils comptent mener, est souvent débattue. Elle n'est pas simple et je voudrais illustrer ce problème sans pour autant prétendre en faire le tour.J'ai ainsi choisi d'illustrer les difficultés par un exemple.

\section{«Le choc des cultures» - une minorité majoritaire}

La constellation des communautés de langue française en milieu minoritaire, pour des raisons historiques, s'est formée et construite de façon «serrée», tout d'abord, au début de son implantation en 
Ontario, pour assurer sa survie. Actuellement ce repliement sur soi-même ne joue plus le même rôle. Il constitue cependant une des caractéristiques des processus de minorisation qui suscitent un jeu entre exclusion et inclusion. Telle est sans doute peut-être l'une des raisons pour laquelle il existe des quartiers «ethniques» dans les grandes villes occidentales - petites Italies et Chinatowns. Les communautés franco-ontariennes n'ont pas échappé à ces mécanismes, bien qu'ils ne se soient pas regroupés en quartiers distincts (Welch 1995). Les gens se connaissent, se co-optent dans les organismes, les corps de métier et les réseaux informels par où passent la connaissance du milieu et les stratégies d'accès aux subventions, aux emplois, etc. Se bâtit alors, sans que cela soit fait de façon nécessairement intentionnelle, une sorte de forteresse qui met le nouveau venu en dehors des cercles de décision ou de partage. Cette situation est souvent vécue très douloureusement par celui ou celle qui est en dehors du cercle, car il ou elle n'a plus de prise sur ce qui se passe. D'où le sentiment d'être exclu, de ne pas être entendu, de ne pas avoir son mot à dire, sentiment relayé bien souvent par les divers préjugés ouvertement exprimés sur telle ou telle origine ethnique, ou même la couleur de la peau.

Pour illustrer ce phénomène insidieux de l'exclusion de «l'Autre», prenons un exemple qui sera peut-être reconnu par beaucoup de lectrices et de lecteurs. Imaginons une réunion entre intervenants et intervenantes rassemblés pour prendre une décision. Il me semble que dans de telles occasions, en Ontario français, la prudence du propos soit récompensée. Les silences, la volonté apparente de concilier les contraires font partie de l'histoire de groupes qui ont souvent vécu en milieu minoritaire. L'expression est feutrée et la confrontation n'est pas de mise. Dans d'autres sociétés, par contre, on a tendance à confronter, à se placer en paradoxe, en particulier quand on est un homme. En France, par exemple, toute l'éducation est tournée vers cette idée que la force des arguments doit se voir et s'entendre... Que se passera-t-il alors, pendant cette réunion, si des personnes venant de ces horizons différents se mettent à discuter? D'un côté, nous aurons, par exemple, une personne avançant ses idées «avec passion»; de l'autre, certaines auront l'impression que cette personne va mettre 
le feu aux poudres et tout faire tomber... Le malaise provoqué par ce comportement inhabituel aboutit à une conversation de sourds, l'un ayant le sentiment qu'on refuse de l'écouter, les autres qu'il essaie de s'accaparer de la parole.

La question qui se pose ici n'est pas tant la «différence» dans la façon de s'exprimer en public que la façon dont est malheureusement résolu le conflit : par un rejet du corps «étranger» qui dérange et / ou par le retrait de la personne qui perçoit bien la cassure qu'elle provoque dans la communication et a ainsi constamment l'impression de «faire des gaffes». À moyen ou à long terme, la personne se rangera sans doute aux habitudes d'ici et s'insérera peut-être dans le groupe majoritaire, à sa façon. Il le

«En participant, par la couleur blanche de sa peau, par son accès à des ressources et à une connaissance très personnelle de sa communauté $e^{5}$ et en ne se posant pas sérieusement la question de ses propres comportements majoritaires, on reproduit les mêmes jeux de pouvoir que ceux que l'on déplore en tant que minorité francoontarienne vis à vis de la majorité anglophone.» faudra bien, si elle veut avoir accès aux services, à l'emploi et si elle veut participer aux décisions qui l'affectent; ce ne sera pas sans difficulté mais elle sera obligée de le faire. Par contre, ceux qui sont dans le cercle, c'est-à-dire ceux qui sont majoritaires dans cette circonstance-ci, ont souvent du mal à voir que leur comportement, leur façon d'être en public ne sont pas «naturels», pas plus que ceux de «l'Autre». En fait, on aura tendance à penser que le retrait ou le rejet a été provoqué par «l'Autre» et par sa façon d'agir. Au meilleur des cas, on déplorera son départ mais on se sentira tout de même soulagé de ne pas avoir à changer soimême. Ainsi, pas plus que l'on ne possède une identité unique et fixée pour toujours, l'on ne reste «minoritaire» dans toutes les circonstances. En participant, par la couleur blanche de sa peau, par son accès à des ressources et à une connaissance très personnelle de sa communauté ${ }^{5}$,et en ne se posant pas sérieusement la question de ses propres comportements majoritaires, on reproduit les mêmes jeux de pouvoir que ceux que l'on déplore en tant que minorité franco-ontarienne vis-à-vis de la majorité anglophone.

Quelles sont les réponses à ces questions? Est-ce que connaître la «culture» de l'autre suffit? Ou n'est-ce pas aussi se poser la question de sa propre culture? Le contact avec l'autre réfléchit, comme en miroir, les normes et les valeurs du groupe majoritaire. C'est dérangeant, en particulier quand on se sent peu sûr de sa propre situation et que la menace d'assimilation se fait sentir. Le 
"...le sens de sa propre marginalité ou de sa minorisation peut être un atout pour penser autrement l'intégration des nouveaux arrivants, dans la mesure où il peut servir de pont à une empathie authentique...» danger est de se raidir et de justifier ses comportements. Cependant, là aussi, le sens de sa propre marginalité ou de sa minorisation peut être un atout pour penser autrement l'intégration des nouveaux arrivants, dans la mesure où il peut servir de pont à une empathie authentique... Cela signifie aussi que l'on a conscience du fait que l'on possède un pouvoir dont on ne soupçonne pas les ramifications. Forger des alliances afin de parer au risque d'assimilation ne suffit pas. Encore faut-il accepter le changement que de telles alliances présupposent.

\section{Conclusions}

Cet article n'a pas cherché à aborder les problématiques propres aux nouveaux arrivants francophones en Ontario dans une perspective systématique de dénonciation des difficultés qu'ils rencontrent dans la vie quotidienne: discriminations au niveau de l'emploi, difficultés d'accès aux services, trop petit nombre de programmes spécifiques à l'accueil des immigrants francophones, malgré la présence grandissante d'organismes qui tentent de répondre à certains de ces besoins. Ce numéro de Reflets, et d'autres articles dans les numéros précédents, témoignent de la naissance graduelle de ces organismes au sein de la francophonie ontarienne. Nous avons préféré analyser, même de façon brève, la façon dont les rapports entre minorités différemment constituées à travers les logiques particulières de l'histoire canadienne peuvent aboutir à certaines impasses, certes, mais aussi devenir la source de création de rapports différents entre population franco-ontarienne née au Canada et nouveaux arrivants francophones.

Il existe donc des impasses qu'il s'agit de relever clairement. Pour en faire le tour, nous reprendrons certaines distinctions faites par Raymond Breton qui s'interroge sur «les conditions qui favorisent l'existence et la vitalité d'une communauté en situation minoritaire», et la rétention de ses membres (1985: 77). L'auteur définit deux types de situation minoritaire. D'une part, un groupe minoritaire peut être «soumis à des restrictions plus ou moins 
sévères quant à leur participation dans les institutions et la vie sociale de l'ensemble de la société» (ibid.). La question qui se pose alors est celle de la mobilité sociale; les membres cherchent à supprimer les barrières qui se lèvent devant leur intégration à la société dominante : ils s'attaqueront ainsi aux différentes sortes de discrimination qui se dressent au sein des organisations et des institutions, aux préjugés individuels, ou encore parieront sur l'apprentissage de la langue et des façons de faire de la majorité. «Il s'agit de faire en sorte que les minoritaires ne soient pas confinés au système social ethnique» qui offre moins de possibilités économiques, politiques et culturelles.

D'autre part, il existe aussi des situations où la participation et l'intégration des membres du groupe minoritaire peuvent se révéler plus faciles. Ils peuvent «traverser les frontières ethniques» de façon plus aisée et ce ne sont plus «les obstacles à la mobilité qui inquiètent, mais l'absence de forces qui retiennent les membres au sein de la communauté minoritaire». Les stratégies de «rétention» des membres de la communauté passeront alors par le renforcement des frontières ethnoculturelles, la reconnaissance interne et externe de sa spécificité et la recherche de sa légitimation politique et institutionnelle.

Bien que la minorité franco-ontarienne puisse se reconnaitre dans ces deux types de situations, il me semble que la distinction posée par Breton nous permet de penser le positionnement actuel des nouveaux arrivants de langue française dans leurs rapports à la communauté franco-ontarienne née au Canada. En effet, les barrières à la mobilité sociale sont plus hautes pour bien des nouveaux arrivants, en particulier la majorité venue des pays du Sud. Les diverses formes de discrimination raciale, s'exerçant à l'égard des personnes de race noire en particulier, l'exigence de l'anglais pour accéder à l'emploi et les conditions (légales) d'arrivée au Canada font que la mobilité au-delà de la frontière de la langue relève davantage de la stratégie de survie que d'une opposition de principe aux revendications des francophones nés au Canada. À cela, nous ajouterons l'idée, développée plus haut, qu'immigrer, c'est aussi et avant tout émigrer, c'est-à-dire avoir un projet de vie axé sur l'amélioration de son sort et/ou celui de sa famille. 
Pour la communauté franco-ontarienne, majoritairement de race blanche, dont les diplômes ou l'expérience de travail sont reconnus et qui s'exprime généralement dans un anglais correct, la question qui se pose relève davantage de la survie collective au sein d'une société de langue anglaise qui s'est historiquement opposée à son développement. Les aspirations des uns et des autres sont toutes les deux légitimes, mais parfois contradictoires. Les tensions et les impasses qui peuvent exister ne relèvent pas nécessairement d'une volonté d'exclusion mais y aboutissent néanmoins objectivement, dans la mesure où les enjeux sont déterminés par différents «positionnements minoritaires» en grande partie dessinés par un pouvoir exercé par le groupe majoritaire, encore aujourd'hui d'origine anglo-saxonne. C'est pourquoi il me semble que toute réflexion sur l'intervention interculturelle en Ontario francophone doit passer par une réflexion et une prise en considération des enjeux politiques posés par la hiérarchisation des rapports sociaux entre groupes minorisés. Une intervention réussie passera, à mon avis, par une prise de conscience, par les intervenantes et les intervenants, de leurs positionnements respectifs dans cette échelle de subordination, construite au nom de la culture mais en fait redevable des rapports sociaux, concrètement et historiquement élaborés au sein même du Canada.

\section{Bibliographie}

APOLLON,Willy (1993). «Fascination et fuite dans la rencontre de l'Autre», Santé mentale au Québec, vol. 18, no 1,7-21.

BADETS, Jane (1993). «Les immigrants du Canada. Dernières tendances», Tendances sociales canadiennes, Statistique Canada, no 29, 8-11.

BARRETTE, Christian, Édithe GAUDET et Denyse LEMAY (1993). Guide de communication interculturelle. Saint-Laurent (Qc), Éditions du renouveau pédagogique.

BEISER, Morton (1988). Puis... la porte s'est ouverte. Problèmes de santé mentale des immigrants et des réfugiés. Rapport du Groupe chargé d'étudier les problèmes de santé mentale des immigrants et des réfugiés au Canada.Ottawa, Santé et Bien-être social Canada.

BER GER, Marie-Josée (1996). «Dilemme des femmes francophones de minorité visible: intégration au marché du travail dans une société ontarienne pluraliste non définie», dans D. Adam (dir. de publication), Femmes francophones et pluralisme en milieu minoritaire. Ottawa, Presses de l'Université d'Ottawa, 7-13. 
BERTOT,J. et A.JACOB (1991). Intervenir avec les immigrants et les réfugiés. Montréal, Québec: Méridien. BIBEAU, G., A. CHAN-YIP, M. LOCK, C. ROUSSEAU et H. FLEURY (1992). La santé mentale et ses visages: un Québec pluriethnique au quotidien, Boucherville, Comité de la santé mentale du Québec, Gaëtan Morin.

BOYD, Monica (1991). «Gender,Visible Minority and Immigrant Earnings Inequality: Reassessing and Employment Equity Premise", Deconstructing a Nation: Immigration, Multiculturalism \& Racism in '90s Canada, sous la dir. de Vic SATZEWICH, Halifax, Fernwood Publishing, 279-321.

BRETON, Raymond (1985). «L'intégration des francophones hors Québec dans des communautés de langue française», Revue de l'Université d'Ottawa, vol 5, no 2, 77-90.

CARDINAL, Linda (1997). L'engagement de la pensée. Écrire en milieu minoritaire francophone au Canada. Ottawa, Le Nordir.

CLARKSON, May et Isabelle DAHAN (1997). La santé, c'est la richesse. Enquête Santé Québec auprès de la communauté du Maghreb et du Moyen-Orient. Monttréal, Santé Québec.

CLARKSON, May et Rosemay EUSTACHE (1997). La santé, c'est la richesse. Enquête Santé Québec auprès de la communauté haïtienne. Monttréal, Santé Québec.

CLARKSON, May et Tran The Nghi (1997). La santé, c'est la richesse. Enquête Santé Québec auprès de la communauté chinoise. Monttréal, Santé Québec.

COHEN-ÉMERIQUE, Margalit (1993). «L'approche interculturelle dans le processus d'aide», Santé mentale au Québec, vol. 18, no 1,69-91.

DAENZER, Patricia (1991). "Unemployment and Minority Immigrants in Canada», International Journal of Sociology and Social Policy, 11: 1-3, 29-50.

DOMINELLI, Lena (1988). Anti-Racist Social Work. London, MacMillan Education Ltd.

ESTABLE, Alam, Mechthilde MAYER et Gordon PON (1997). Teach Me To Thunder. Ottawa.

GILBERT, Anne et André LANGLOIS (1994). Regard sur les nouvelles réalités franco-ontariennes. Les Francophones tels qu'ils sont, Troisième édition.Vanier,Association canadienne-française de l'Ontario.

GROUPE DE TRAVAIL SUR LES FEMMES IMMIGRANTES ET LA VIOLENCE CONJUGALE (1997). Les femmes immigrantes et la violence conjugale: pour un véritable accès à l'exercice de leurs droits. Montréal, Maison Flora Tristan.

HENEMAN, Bernard et al. (1994). Adéquation des services aux jeunes familles immigrantes, Université de Montréal - École de service social et Direction de la santé publique, Régie régionale de Montréal Centre.

HYMAN, Ilene, Morton BEISER et NHIVU (1996). «The Mental Health of Refugee Children in Canada», Refuge, vol. 15, no 5, 4-7.

JACOB, André (1992) «Services sociaux et groupes ethnoculturels: le débat et les pratiques au Québec», Nouvelles Pratiques Sociales, vol. 5, no 2,37-53.

JUTEAU-LEE, Danielle (1980). «Français d'Amérique, Canadiens, Canadiens-Français, FrancoOntariens, Ontarois: qui sommes-nous?». Pluriel, no 24, 21-42.

JUTEAU, Damielle (1986). «L'État et les immigrés: de l'immigration aux communautés culturelles», dans Guillaume, P., R. Pelletier, J-M. Lacroix et J. Zylberberg (dir. de publication) Minorité et État. Québec, Centre d'études canadiennes de la Maison des sciences de l'homme d'Aquitaine et LÉPA de l’Université Laval, 35-50. 
KÉRISIT, Michèle (à paraître). «Intervenir auprès des familles immigrantes: Quelques points de repère", dans M.B. Tahon (dir. de publication) Famille et fragmentation. Presses de l'Université d'Ottawa.

LABELLE, Micheline (1990). «Femmes et immigration au Canada: bilan et perspectives», Études ethniques au Canada, vol. 22, no 1, 67-82.

LEGAULT Gisèle et Myriam LAFRENIÈRE (1992). «Situations d'incompréhensions interculturelles dans les services sociaux: problématiques», Santé mentale au Québec, vol. 17, no 2, 113-133.

LEGAULT, Gisèle (1993). «Femmes immigrantes: problématiques et intervention féministe», Service social, 42: 1, 63-80.

LOCKE, Don C. (1992). Increasing Multicultural Understanding: a Comprehensive Model. Thousand Oaks, London \& New Delhi, Sage Publications.

MACLEOD, Linda et MariaY.SHIN (1994). Like a Wingless Bird. A Tribute to the Survival and Courage of Women Who Are Abused and Who Speak Neither English Nor French, Ottawa, National Clearinghouse on Family Violence.

MASI, Ralph (1993). Health and Cultures: Exploring the Relationship. Vol 1: Policies, Professional Practice and Education.Vol 2: Programs, Services and Care. Toronto, Mosaic Press.

MCKAGUE, Ormond (1991). Racism in Canada. Saskatoon, The Fifth House Reader.

MILLS, Megan Stuart (1993) «Mental Health Resilience of Refugees: the Case of Tamil Refugees», Refuge, vol. 13, no 3, 27-31.

MORO, Marie Rose (1993). «Principes théoriques et méthodologiques de l'ethnopsychiatrie. L'exemple du travail avec les enfants de migrants et leurs familles», Santé mentale au Québec, vol. XVII, no 2, 71-98.

RIDLEY, Charles R. (1995). Overcoming Unintentional Racism in Counseling and Therapy. A Practioner's Guide to Intentional Intervention. Thousand Oaks, London \& New Delhi, Sage Publications.

VATZ LAAROUSSI, Michèle, Diane LESSARD, Maria Elisa MONTEJO et MonicaVIANA (1996). Femmes immigrantes à Sherbrooke: modes de vie et reconstruction identitaire, Rapport présenté au Conseil québécois de recherche sociale. Collectif de recherche sur les femmes et le changement, Université de Sherbrooke.

WELCH, David (1995). «Les Franco-Ontariens : la résistance comme mode de vie», Reflets, vol 1, no $1,20-42$.

WELCH,David (1993). «Early Franco-Ontarian Schooling as a Reflection and Creator of Community Identity», Ontario History, vol. LXXXV, no 4, 321-347.

\section{Notes}

1. Cet article reprend en partie une conférence intitulée Pratiques interculturelles en milieu minoritaire, prononcée au Colloque annuel du Comité francophone de l'Association professionnelle des travailleurs sociaux de l'Ontario. Université d'Ottawa, avril 1995.

2. Ces appellations sont contestées et à juste titre. Les termes 
«de souche» ou «immigrants» perpétuent l'idée d'une prééminence de la notion de territorialité par rapport à la citoyenneté. Le terme «ethnoculturel» a été particulièrement contesté dans la mesure où il ne reflète pas les inégalités structurelles qui existent dans les rapports entre Canadiens d'origine française ou britannique et personnes qui ont immigré d'autres pays que ceux-là. Le terme de «minorité visible» reporte sur la couleur de la peau des traits qui n'ont rien à voir avec celle-ci. Néanmoins, à mon avis, cette appellation a au moins le mérite de signaler l'importance qu'a prise, dans les sociétés blanches, occidentales, et canadienne en particulier, la distinction de la couleur de la peau pour signifier la différence, et construire et maintenir les inégalités sociales.

3. Se posant la question de savoir qui est immigrant et qui ne l'est pas, Danielle Juteau (1986: 37) résume ainsi la question de certains termes utilisés: «Il existerait au Canada trois catégories de personnes: celles qui sont nées à l'étranger, celles dont les ancêtres sont immigrants, et celles dont les ancêtres ne sont pas immigrants. Cette activité de catégorisation n'est pas sans surprendre, puisque nous sommes tous, en quelque sorte, filles et fils d'immigrants. Elle exprime néanmoins, au niveau symbolique, le statut concret des catégories sociales en place. Ce statut concret renvoie, quant à lui, au modèle de séquence (colonisation, immigration) et aux rapports inégalitaires qui en découlent».

4. «Ces gens-là» est une expression souvent utilisée en Ontario français pour désigner «les Autres», en particulier les immigrants. L'utilisation de cette expression péjorative, en français standard, a pour effet d'exclure les nouveaux arrivants de l'expérience et de la société franco-ontariennes.

5. L'une des caractéristiques des minorités est la force des réseaux personnels, force qui témoigne de la volonté de se reconnaître comme groupe et est, dans une certaine mesure, une stratégie de survie. Elle est cependant l'un des obstacles les plus forts à l'intégration des «minorités» au sein d'une «minorité». 